Supplementary information

\title{
Shape-Control of ZnTe Nanocrystal Growth in Organic Solution
}

Jun Zhang ${ }^{1}$, Kai Sun², Amar Kumbhar ${ }^{3}$ and Jiye Fang*1

${ }^{1}$ Department of Chemistry, State University of New York at Binghamton, Binghamton, NY 13902

${ }^{2}$ Electron Microbeam Analysis Laboratory, University of Michigan, Ann Arbor, MI 48109

${ }^{3}$ Electron Microscope Facility, Clemson University, Anderson, SC 29625

Email: jfang@binghamton.edu 


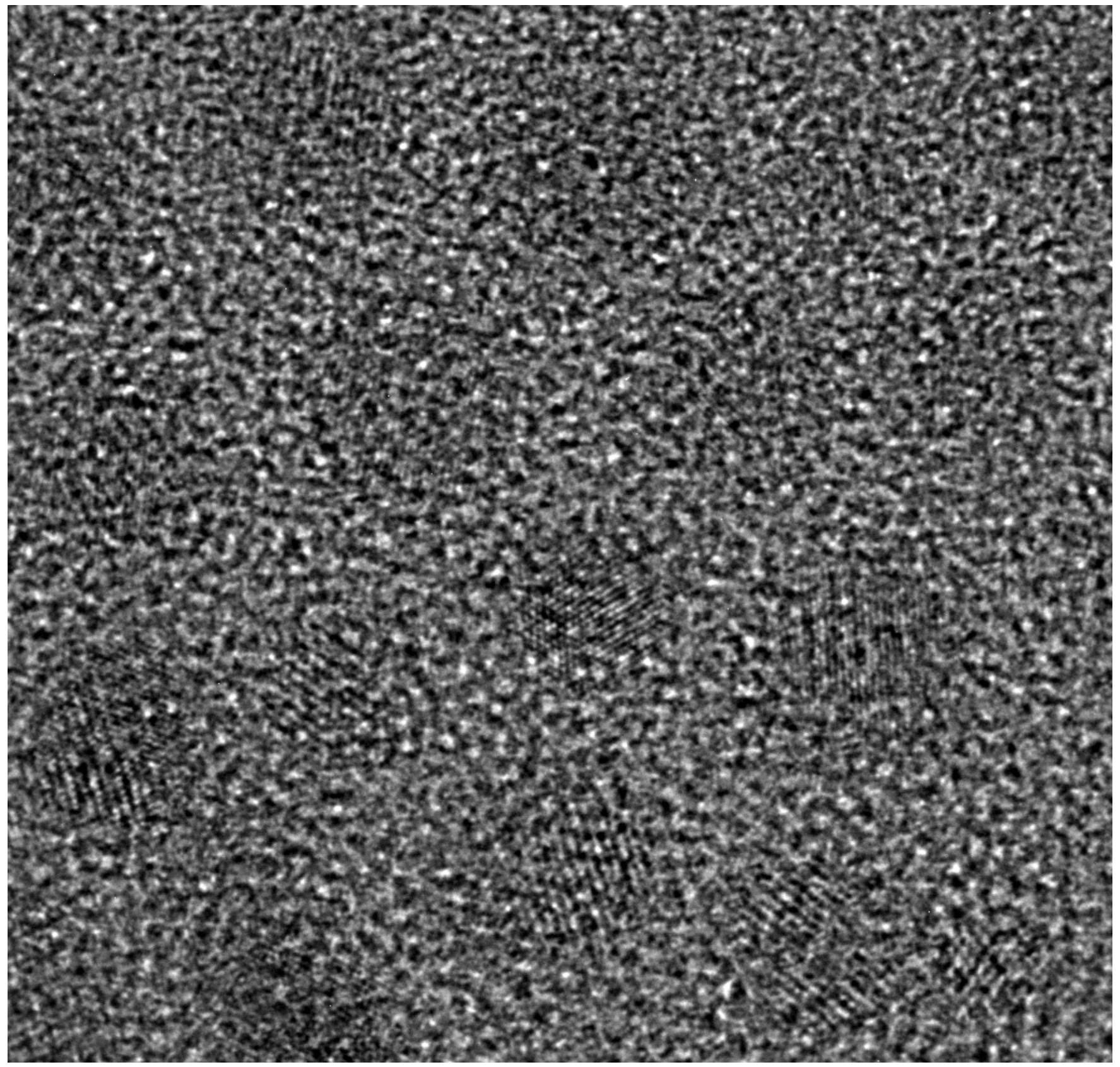

Fig. S1. High-resolution TEM image of ZnTe quasi-spherical nanocrystals synthesized from pathway of Reaction I. 


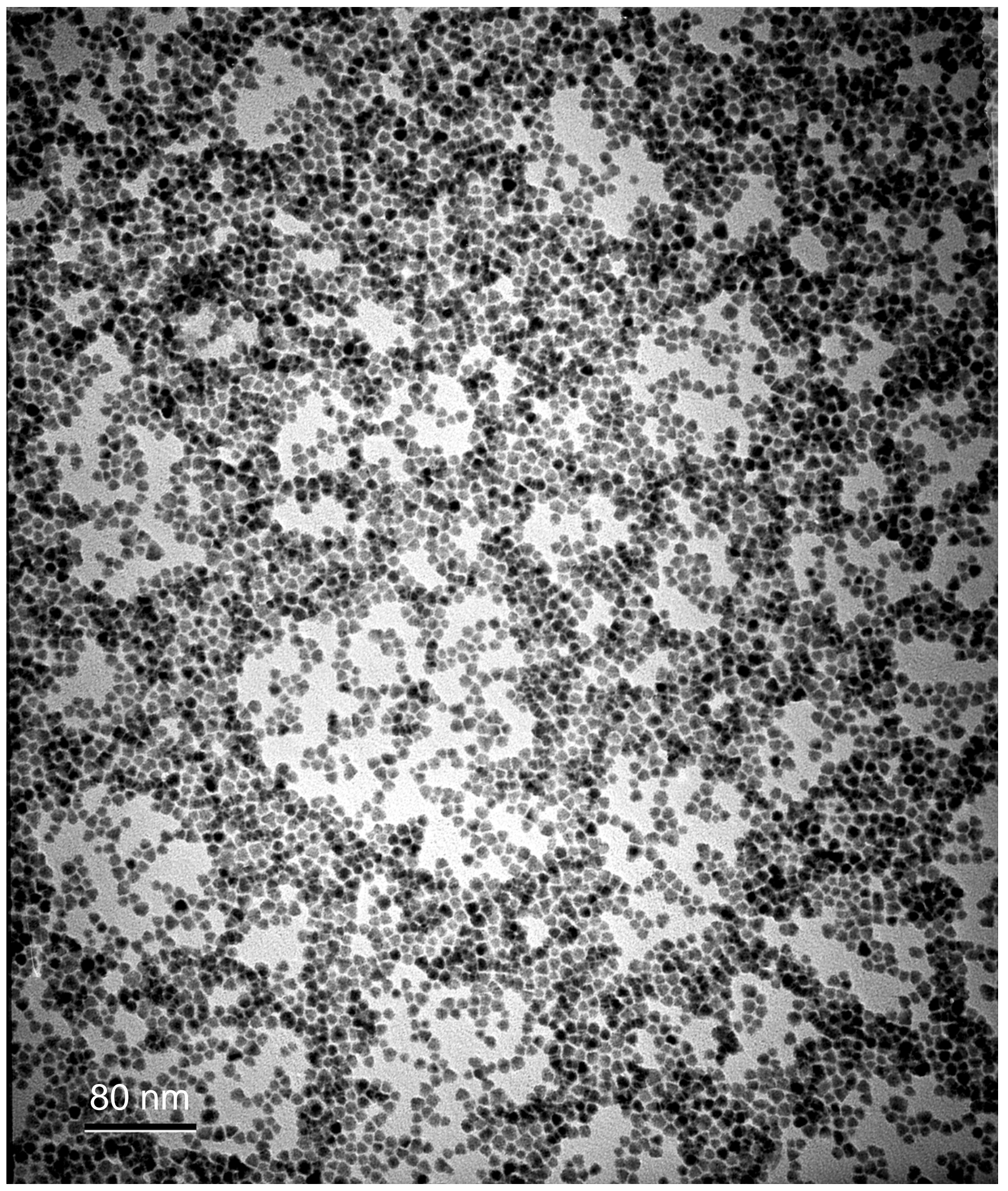

Fig. S2. TEM image of ZnTe tetrahedral nanocrystals synthesized from pathway of Reaction II. 


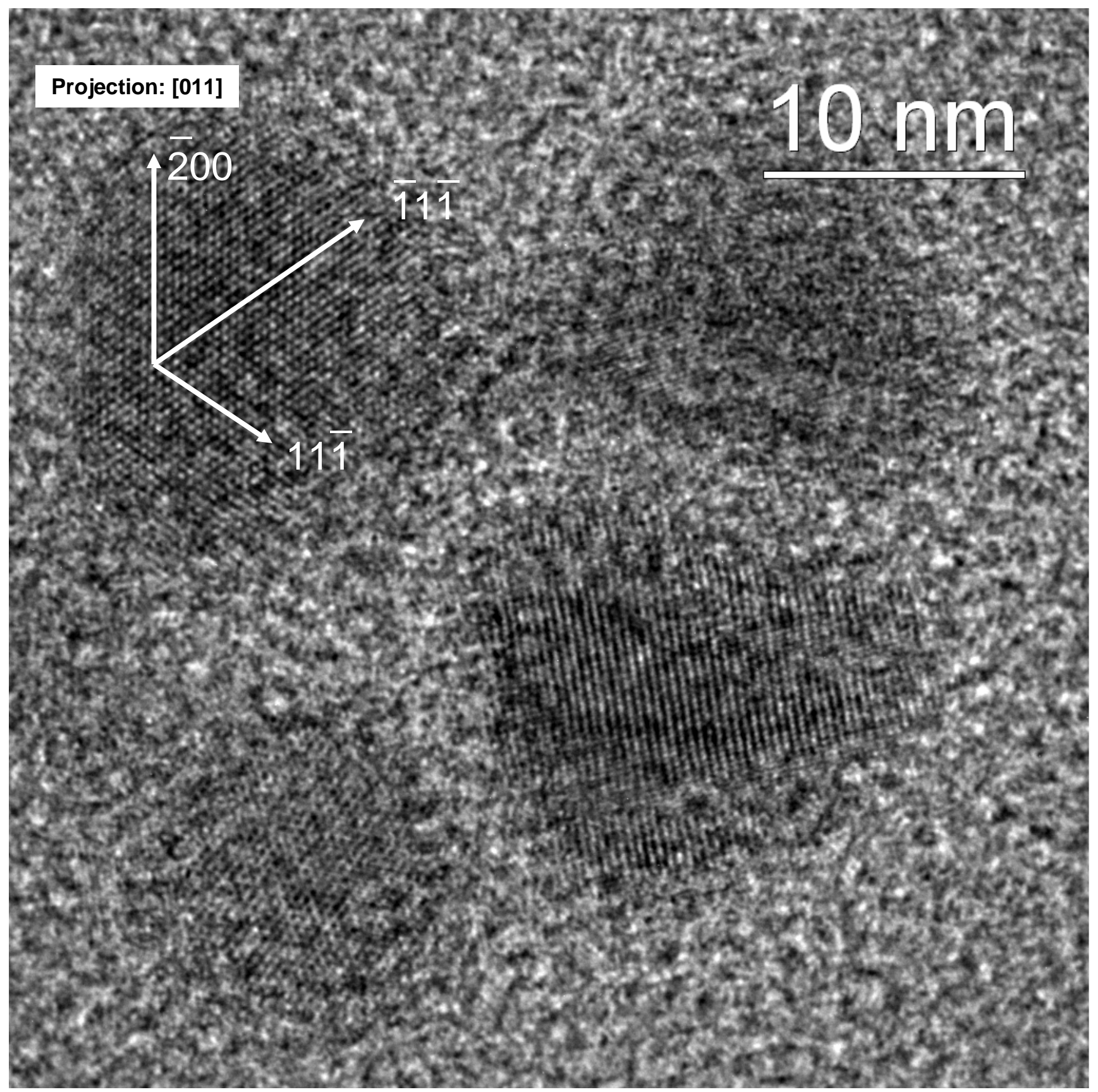

Fig. S3. High-resolution TEM image of ZnTe tetrahedral nanocrystals synthesized from pathway of Reaction II. 


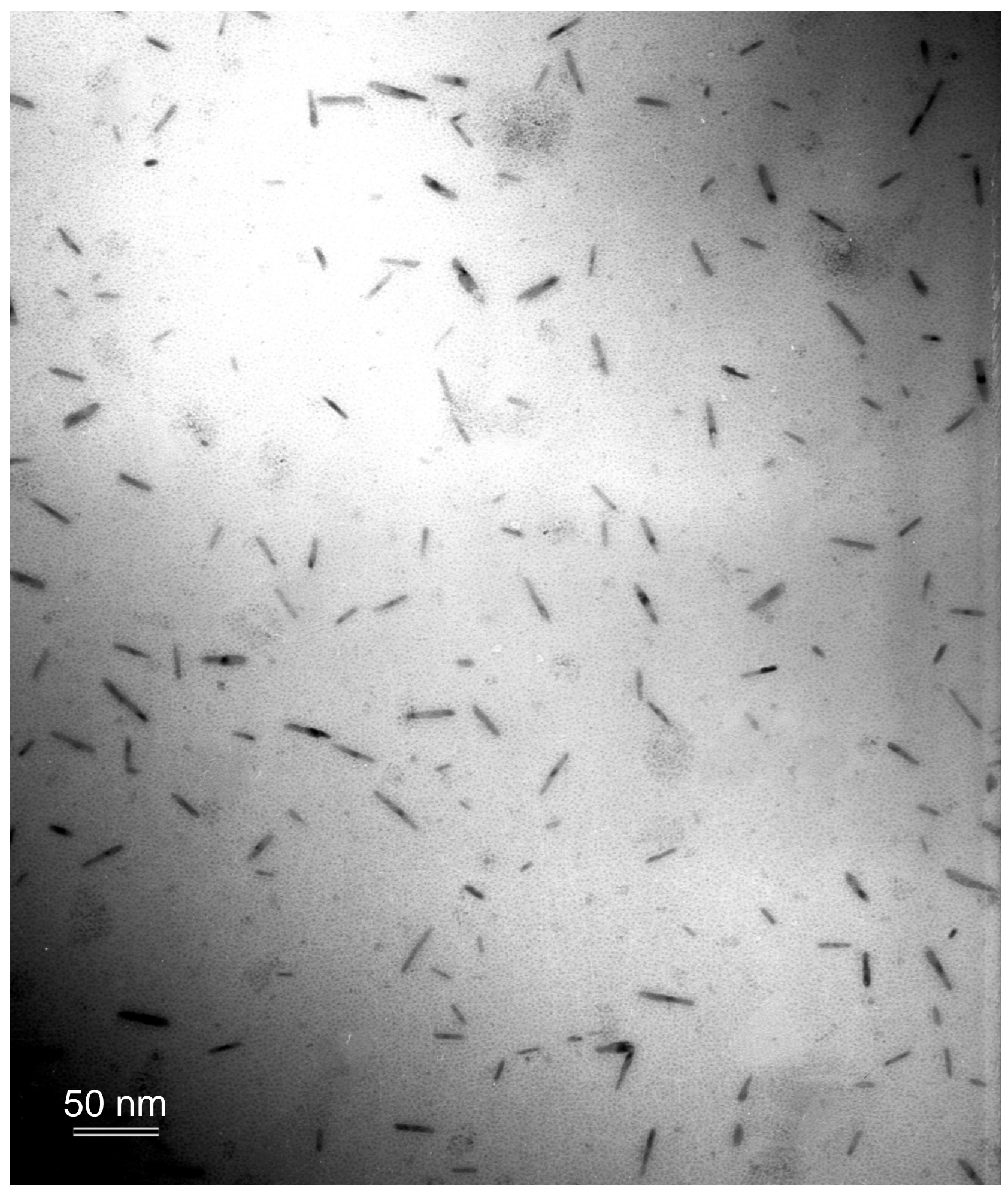

Fig. S4. TEM image of ZnTe nanorods synthesized from pathway of Reaction III. The precursors as described in the context were aged for $24 \mathrm{~h}$. 


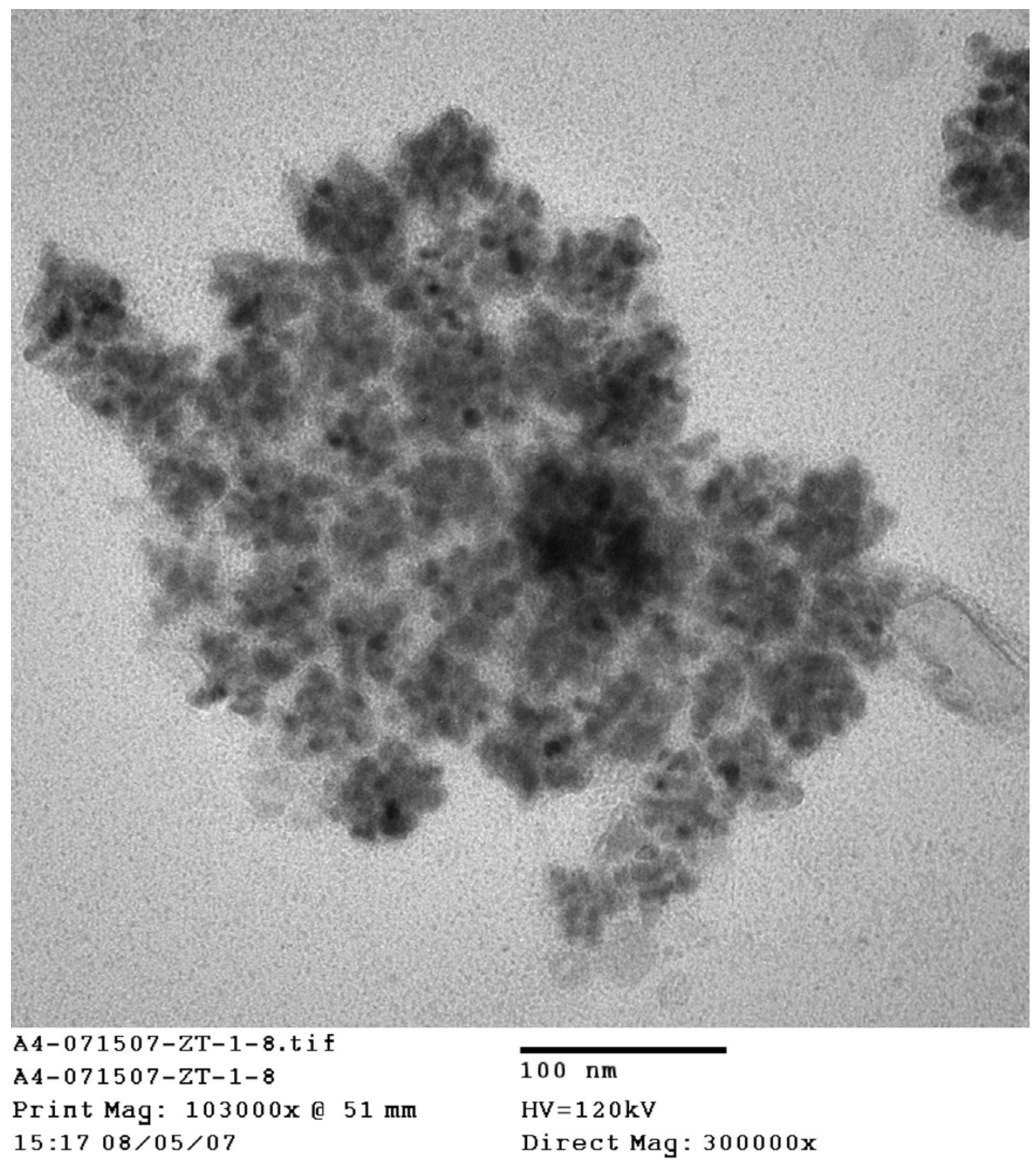

Fig. S5. TEM image showing agglomeration of quasi-spherical ZnTe nanocrystals synthesized from pathway of Reaction I. The growth time is $1 \mathrm{~h}$. 